PHYSICAL REVIEW D 95, 129903(E) (2017)

\title{
Publisher's Note: Reconsidering seismological constraints on the available parameter space of macroscopic dark matter [Phys. Rev. D 95, 063006 (2017)]
}

David Cyncynates, Joshua Chiel, Jagjit Sidhu, and Glenn D. Starkman (Received 7 June 2017; published 15 June 2017)

DOI: $10.1103 /$ PhysRevD.95.129903

This paper was published online on 7 March 2017 with a conversion error in Eq. (A6). Equation (A6) should read as $\dot{r}_{\mathrm{m}}(t) \approx \sqrt{\frac{\alpha}{t} \ln [C / t]}$. The equation has been corrected as of 7 June 2017. The equation is incorrect in the printed version of the journal. 